\title{
Covid-19 : Natural immunity versus vaccine immunity
}

\author{
Helene Banoun
}

Funding: The author(s) received no specific funding for this work.

Potential competing interests: The author(s) declared that no potential competing interests exist.

\section{Abstract}

It should not be necessary to write an article to demonstrate the superiority of natural immunity over vaccine immunity, but it is necessary in view of the many underhanded attacks that tend to make artificial immunity seem more effective. Furthermore, it is officially recommended to vaccinate people who have already been infected with Covid-19.

Survivors of the 2003 SARS-CoV have cellular immune memory more than 17 years after infection. Numerous studies have explored humoral (antibody) and cellular immunity to SARS-CoV-2 after Covid-19 infection: it persists for at least one year (and even 14 months) in a robust manner in convalescents and would be of better quality than vaccine immunity: natural antibodies are more potent, have a broader spectrum, and are able to evolve against variants more efficiently than vaccine antibodies.

These in vitro studies are confirmed by the protection against reinfections conferred by a primary infection, particularly in early and highly vaccinated countries such as Israel and the United Kingdom. Vaccination of a primo-infected person could also decrease the effectiveness of his natural immunity against future reinfections.

Neutralizing antibodies may not be the correct correlate of protection against infection, as high levels of neutralizing antibodies have been found in patients with severe Covid, and vaccinated patients, who are more susceptible to reinfection than primary patients, have antibody levels equivalent to or higher than those observed in primary patients.

The neutralizing capacity of these antibodies directed against the membrane spike protein is measured in vitro. This neutralizing capacity may be different in vivo; it may be diminished by the presence of antibodies that facilitate infection; and antibodies are produced against other viral antigens during natural infection. Cellular immunity could be the right correlate of protection and this type of response could be of lower quality in vaccinated subjects.

In addition, vaccination of convalescent subjects could be risky: more systemic adverse events are observed in convalescent subjects than in naïve subjects after the first dose of vaccine. Vaccination may decrease the ability to respond to future variants. It could also have a non- specific effect of remodeling the innate immune response by decreasing the potential response to other viruses or cancers and by modifying the course of inflammatory and autoimmune diseases. 
The natural immunity of a population should therefore be explored before proposing vaccination: up to $50 \%$ of the population may have been infected in some countries.

\section{Introduction}

It should not be necessary to write this article to demonstrate that natural immunity is superior to vaccine immunity: indeed, since the appearance of life on Earth, natural immunity has been developing in all living beings in response to predators and all this without vaccines! Innate immunity preceded adaptive immunity and allowed us to recognize foreign pathogens, eliminate them and maintain an immune memoryl.

Until June 2020, innate immunity, related to the human innate and adaptive/acquired immune system, was recognized by the WHO as a key component of herd immunity to novel pathogens.

Until November 2020, the WHO admitted that immunity can be provided by infection or by vaccination, on November 13, 2020, the WHO affirms that herd immunity of a population can only be achieved by vaccination and not by natural infection. This is reaffirmed on December 31, 2020 for Covid-19, while recognizing that we do not know the threshold of vaccine coverage to be obtained to achieve this herd immunity. (only the December 31, 2020 definition is still accessible, https://www.who.int/news-room/q-adetail/herd-immunity- lockdowns-and-covid-19, the previous ones are visible on the screenshot below) In a recent publication, the John Hopkins Institute only mentions vaccine immunity in a discussion about the hypothetical herd immunity to be expected against Covid2

\section{Immunity to coronaviruses}

The knowledge on the immunity acquired after a coronavirus infection is very insufficient. In 1990, the only in vivo human experiment on acquired immunity to common cold coronaviruses was published3 : volunteers were infected with coronavirus 229E, which causes cold symptoms, and returned one year later to be re-exposed to the virus. Most people were re-infected, but those who had been sick the previous year did not develop cold symptoms. The study authors also reported that the period of virus shedding, when a person is infectious, was shorter in those who had been infected the previous year. For 2003 SARS-CoV-1, cellular immunity has been shown to persist for 11 years according to a 2016 study4.

And according to a 2020 study, patients who survived the 2003 SARS outbreak have memory cell reactivity 17 years after the outbreak5.

Because SARS-CoV-2 causing Covid-19 is a closely related virus to 2003 SARS-CoV-1 and shares many similarities in epidemiology and pathogenicity, it is possible that cellular immunity to the Covid-19 virus could persist for years.

\section{Immunity measured in vitro after infection with SARS-CoV-2}

Studies comparing in vitro (antibody assay, neutralizing activity, memory B and T cells) natural and 
vaccine immunity seem to show superiority of natural immunity. It seems that vaccination modifies the immune response of convalescents and not necessarily in a good way: it could reduce the subsequent capacity of convalescents to react to future variants. Publications (examples below) show that neutralizing antibodies and memory B (humoral immunity) and $\mathrm{T}$ (cellular immunity) cells persist for at least one year in convalescents. Protection against SARS-CoV-2 infection could last for years6.

\section{Humoral immunity (antibodies)}

It is normal to observe a drop in circulating antibody levels after an infection, as happens with all infections, otherwise the blood would be thickened by all the antibodies that accumulate over a lifetime. At the same time, the immune memory is built and refined.

"The slight decrease in antibodies observed in convalescent individuals does not reflect a real decline in humoral immunity, but rather a contraction of the immune response, while maturation of antibody affinity occurs and anti-Spike memory B cells persist. "7

Several publications suggest that natural immune memory is even better than vaccine memory. According to Cho et al. 8, natural antibodies are more potent and broader spectrum than vaccine antibodies. As discussed below, the neutralizing power of plasma measured in vitro is unlikely to be evidence of protection against infection.

Antibodies to the RBD (receptor binding domain) of the spike protein and to the whole spike persist for at least 1 year after recovery from Covid, and the level of anti-RBD antibodies correlates with the level of neutralizing antibodies9.

In vaccinated individuals, antibody levels decrease by $40 \%$ per month and by less than $5 \%$ per month in convalescents10.

\section{Cellular immunity}

In recovered COVID-19 patients, SARS-CoV-2-specific CD8+ T-cell responses remained largely intact and could recognize virtually all mutations of the variants studied11.

In dialysis patients, a German team found better humoral and cellular immunity in convalescents compared with vaccinated patients: this relates to neutralizing antibody levels and T-cell responses directed against the spike protein. Levels of CD4+ S-reactive T cells, helper T cells, and memory effector cells are higher in convalescents than in vaccinees12. Most COVID-19 patients induce a broad-based immune defense against SARS-CoV-2 infection, encompassing antibodies and memory B cells recognizing both the RBD and other regions of the spike, broadly specific and polyfunctional CD4+ T cells, and polyfunctional CD8+ T cells. The immune response to natural infection is likely to provide some degree of protective immunity, even against SARS-CoV-2 variants, because CD4+ and CD8+ T-cell epitopes are likely to be conserved13.

According to a meta-analysis of 18 studies of SARS-CoV-2 epitopes recognized by T cells of Covid-19 convalescents: The high diversity of epitopes not confined to the S protein does not appear to be affected by VOC variants, at least before the arrival of the delta variant14.

A recent Israeli publication of September 21, 2021, shows the persistence of humoral immunity in 
convalescents for 14 months, which is not the case in vaccinated individuals. Bypass of innate immunity by mRNA vaccines could affect the ability of the immune system to maintain sufficient levels of long-lived plasma cells15.

\section{Protection against reinfection by infection acquired immunity}

These in vitro observations of antibody and memory cell levels are well confirmed by looking for reinfections in convalescents: these are very rare.

Numerous publications (see for bibliography the recent BMJ article16) show that the rate of reinfection is very low (less than 1\%) following a first infection with SARS-CoV-2. The vast majority of these reinfections are not reinfections as such since they are asymptomatic: they are simply nasal carriage of virus without systemic infection. This is not surprising for a cold virus; the nose is an immune sanctuary where blood antibodies do not circulate.

\section{What is the proportion of people in the world who are naturally immune to infection?}

There is patchy information: in Kenya in November 2020, more than 1/3 of the population was HIV positive17 ; in India in January $2021,25 \%$ of the population was seropositive18. In China, in Wuhan, the epicenter of the epidemic, a very low rate of seropositivity has been found (less than 4\% in April 202019). Infected persons with few or no symptoms do not develop this type of SARS-CoV-2 specific antibodies, they would have been protected by their cross immunity to common cold coronaviruses 23 .

In France, the HAS estimates that $23 \%$ of the population has been infected while only $8 \%$ have been detected by PCR or antigenic test20.

It is estimated that almost half of all Americans have been infected with SARS-CoV-216

By August 2020, 40\% of the Malagasy population (estimated from blood donors) would have been seropositive21.

One obstacle to these seroprevalence studies is that serological tests lack standardization22 .

\section{Another problem is that one can be immune and not have antibodies (anti-S or other antibodies).}

According to publications from 2020, people who are infected but have not developed symptoms have a T cell reaction but no antibodies23 (for a review); this is confirmed in 2021 : Le Bert et al. 24 search seropositive asymptomatics for anti-NP-IgG antibodies (antibodies to the virus nucleocapsid): all seropositives have a highly functional virus-specific cellular immune response. In addition, individuals who are anti-NP IgG negative also have T cells that react specifically with SARS-CoV-2 antigens.

\section{What is a reinfection?}

The CDC defines reinfection as a positive test regardless of symptoms (and unvaccinated people are tested more than vaccinated people, due to the health pass)25. The only studies that claim that infection does not protect against reinfection do not clearly define the term reinfection: a positive PCR with a Ct of 35 (low viral load or false positive) without symptoms cannot be defined as reinfection.

The study of reinfections shows that infection provides at least as much protection against infection and probably more than vaccination. 
As early as April 2021, F Krammer proposed that natural immunity was better than vaccine immunity, based on a British study comparing primary infected and vaccinated healthcare workers 26 .

Since this publication, the continuation of this SIREN study has concerned reinfections with the delta variant and shows the superiority of natural immunity 27 . Of the 9,831 healthcare workers who were already infected with Covid before the study began, 51 were reinfected between April and July 2021, the period of delta variant circulation. Among these 51 persons, 45 were vaccinated since at least 21 days after dose 1 or 14 days after dose 2, nothing is specified about the 6 remaining persons: either they were not vaccinated or, they were infected in the few days following dose 1 or 2 because of the ADE (antibody dependent enhancement $=$ facilitation of infection by vaccine antibodies): why is it not specified? All in all, infection protects against reinfection, which is not the case with vaccination According to a French study, the relative reduction in the incidence of reinfection by SARS- CoV- 2 is $96.7 \%$ in convalescents28 compared with non-infected people.

In young army recruits, reinfections (in HIV-positive individuals) are 5 times rarer than infections in seronegative individuals, and reinfected individuals are most often asymptomatic29.

Covid epidemic in a gold mine in French Guiana with gamma variant, June 202130

Key observations: Covid recoveries are not infected (unless they were vaccinated), 15 out of 25 vaccinated are infected (recovered or not), unvaccinated are infected last, so it is possible that the vaccinated have infected the unvaccinated. The vaccinated have a slightly higher viral load than the unvaccinated. No severe form is observed despite the strong presence of risk factors in infected minors.

And finally, we must of course mention the studies carried out in Israel, the country vaccinated the earliest on a large proportion of the population. Gazit et al. (Israel) 31 reported that never-infected individuals vaccinated against SARS-CoV-2 had a 13.06-fold increased risk of infection with the Delta variant (or 5.96fold depending on the method of calculation) compared with previously infected individuals. The increased risk was also significant for symptomatic disease. Compared with primary infected individuals, vaccinated individuals not infected with SARS-CoV-2 had a 27-fold increased risk of symptomatic infection (or 7-fold increased risk by calculation) of symptomatic disease. Vaccinated individuals also had a higher risk of COVID-19-related hospitalizations (6.7-fold) than previously infected individuals. This study demonstrated that natural immunity confers longer-lasting and stronger protection against infection, symptomatic illness, and hospitalizations caused by the SARS-CoV-2 Delta variant, compared with immunity induced by the two-dose BNT162b2 vaccine. Those previously infected with SARS-CoV-2 who received a single dose of the vaccine gained additional protection against the Delta variant.

It should be added, of course, as usual, that post-vaccination Covids are excluded since, in infected and vaccinated individuals, they are only counted from 7 days after the injection. The ADE is not taken into account, so the results may be unfavorable to vaccination in the infected, we can't know!

On the other hand, people who were infected before February 28, 2021 are excluded, so basically the elderly and frail people (vaccinated first from December 2020): the result would be even more unfavorable to the vaccine if they were included. 
This study was reported in Science Mag and summarized as follows: Israelis who had an infection were more protected against the Delta variant of the coronavirus than those who received a COVID-19 vaccine32.

Meta-analysis of natural immunity data (September 21, 2021)33

Natural immunity is equivalent to or superior to vaccination in protecting against Covid: no study concludes that vaccine protection is superior to natural immunity with statistical certainty, but observational studies have confirmed the benefit of protection by natural immunity.

The benefit of vaccinating people who have already contracted Covid is zero or very small ( 3 out of 4 studies find no benefit of vaccinating convalescents and the additional protection found by pooling all studies is $0.004 \%)$.

\section{Vaccine immunity is classically associated with the titer of neutralizing antibodies in vitro, but are neutralizing antibodies the right correlate of protection?}

There are conflicting results on the persistence of neutralizing antibodies after infection: in some studies these antibodies persist for at least one year, in others their level decreases rapidly, perhaps due to the methods used, which differ from one study to another?

To measure the neutralizing power of a serum in vitro, these studies use either live viruses cultured on different types of cells, or reconstituted pseudoviruses carrying mutated spike proteins of SARS-CoV-2 on their surface.

According to a meta-analysis34, the levels of neutralizing antibodies induced by mRNA vaccines against SARS-CoV-2 variants were similar to or higher than those obtained from naturally infected individuals. This is not consistent with the low rate of reinfection in primary infected versus vaccinated individuals, as seen above in the United Kingdom27 reinfections with the delta variant in health care workers are only in fully or partially vaccinated individuals.

According to numerous studies, the level of neutralizing antibodies and their spectrum against the variants is significantly increased after vaccination in previously infected individuals35. According to Stamatos36, vaccination can increase the level of neutralizing antibodies against variants in primary infected individuals up to 1000 -fold. According to Reynods et al. 37, naive vaccinees have a similar antibody response to that of naturally infected individuals but T cell responses are more limited and sometimes absent.

On the contrary, according to Havervall et al. the levels of neutralizing antibodies to wild-type and mutant strains 12 months after natural infection are lower than in vaccinees38.

According to Planas et al.39 convalescent sera lose their ability to neutralize the delta variant in vitro over time. A single dose of vaccine yields almost no neutralizing antibodies to the delta variant, regardless of the vaccine.

In summary, vaccination would result in higher levels of neutralizing antibodies than natural infection, which contradicts the different rates of reinfection observed between infected and vaccinated individuals: are neutralizing antibodies the right correlate of protection? According to Prof. La Scola in a video, the presence of high levels of neutralizing antibodies in vaccinated 
individuals does not prevent them from being infected40. There is a correlation between antibody levels measured by Elisa and serum neutralization but no correlation between in vitro neutralizing antibody levels and protection against infection: "In this study, $5 \%$ of the vaccinated individuals became infected, compared with only $0.23 \%$ of those who

had Covid-19 disease, so the effect of cellular immunity is not objectified by serum neutralization; indeed, most of the vaccinated individuals have a high level of antibodies. This is confirmed by other publications 16.

The neutralizing Ac acquired after infection varies greatly depending on the variant that caused the infection; mRNA vaccines induce higher levels of neutralizing antibodies than the infection41 and yet the infection protects better than the vaccine and severe Covid patients may have high levels of neutralizing antibody.

The theoretical explanation for this lack of correlation between in vitro neutralizing antibodies and protection could be found here: virus neutralization is studied in vitro, under conditions totally different from what happens in vivo, and may not reflect what happens in the body 42

\section{Immune memory concerns other epitopes than those of the spike}

Almost all of the studies concern anti-spike antibodies, however, "The majority of epitopes targeted by CD8+ are found in the internal proteins of the virus or in the NSPs [non-structural proteins, which do not include the spike protein]"43

Protection from infection is likely due as much to immune memory as to persistence of high antibody levels44

\section{Neutralizing antibodies may lose their properties in the presence of facilitating antibodies produced by vaccination}

The present article cannot elaborate on this subject, which has been discussed in recent publications 45

The role of mucosal immunity, which is stimulated by natural infection and not by IM vaccination, should also be mentioned: "Almost all studies of the immune response in COVID-19 have focused exclusively on serum antibodies and systemic cell-mediated immunity, including innate responses. Mucosal immunity and secretory and circulating IgA antibodies play an important role in COVID-19, and it is important to elucidate this in order to understand in particular the asymptomatic and mild states of infection, which appear to represent the majority of cases. "46

"Vaccination of never-infected persons may not induce strong humoral immunity in the respiratory mucosa, because neutralizing antibodies to SARS-CoV-2 are rarely detected in nasal swabs from vaccinated persons. If it turns out that current vaccination strategies fail to elicit robust humoral and cellmediated immune responses in the respiratory tract, then strategies to better elicit SARS-CoV-2-specific B and T cells in the mucosa of never-infected individuals should be considered-for example, by implementing intranasal mRNA immunization, which is more likely to induce sterilizing immunity. "47

Protection against Covid may also be due to cross-immunity to common cold coronaviruses 23 . A recent study shows that children have SARS-CoV-2 reactive cells in their tonsils, even though they have 
not been exposed to the virus, so pre-existing cellular cross-immunity may explain their resistance to Covid48.

\section{Is it appropriate to vaccinate Covid convalescents?}

The case of long Covids49

Two theories explain long covids: either the virus persists and causes chronic inflammation, or it is autoimmunity through the production of autoantibodies against viral proteins with similarities to human proteins. Vaccination can aggravate this phenomenon but also improve it, we do not know this in advance. mRNA vaccines aberrantly direct T-cell responses in patients with neurological sequelae of Covid infection (Covid long): is it appropriate to vaccinate them?50

\section{Adverse effect of vaccine on ability to respond to new variants}

Vaccination of convalescent subjects may be risky: convalescent subjects have more systemic adverse events than naïve subjects after the first dose of vaccine.51 In addition, vaccination does not improve the ability to respond to new variants and may even be harmful.

One study found somewhat contradictory results: vaccination increased humoral and memory response in convalescents by 30 to 50 times. The neutralizing activity in unvaccinated convalescents is weakened against the variants (the delta variant is not tested) but increased after vaccination in convalescents. The neutralization spectrum of antibodies collected in convalescents broadens with time and after one year the potency and neutralization spectrum are increased against the variants Vaccination does not broaden the spectrum of clonal expansion compared to unvaccinated convalescents after one year. RBDbinding antibody affinity is not increased by vaccination after one year, no difference in neutralizing effect is observed.52

\section{The second dose of BNT162b2 vaccine results in a reduction in cellular immunity in persons} who have recovered from COVID-19, suggesting that a second dose, according to the current standard vaccination regimen, may not be necessary in persons previously infected with SARS-CoV-2: the second dose of vaccine not only does not enhance humoral immunity, but also determines a contraction of the spike-specific T-cell response.53

The natural immunity of the infected is hampered by a subsequent anti-Covid vaccination Vaccination of convalescents reduces the ability to produce broad-spectrum antibodies against future variants; unvaccinated convalescents have this ability. 54

The affinity of natural antibodies increases more with time than that of vaccine antibodies After vaccination of primary patients, the level of neutralizing antibodies increases, but not the potency of these antibodies or the ability to evolve to broad-spectrum protection thereafter. This is also true for the delta variant.

The difference in memory cell compartments in response to infection or vaccination (mRNA) is consistent with the greater level of protection conferred by natural infection with the variants. A relative reduction in reactivity of sera with the B.1.1.7 variant compared to the Wuhan-1 variant is seen after the second booster immunization. These data allow comparison of the different vaccines in terms of anti-S antibody production and call into question the value of repeated immunization with the same $\mathrm{S}$ 
protein sequence. In convalescents, vaccination caused a relative loss of recognition of the B.1.1.7 variant beginning as early as 15 days after the first dose of Pfizer and worsening thereafter55.

The CD4+ T cell response is decreased in convalescents after vaccination compared to naïve individuals: vaccination is thought to damage the $T$ cell response. After vaccination, the magnitude of the immune response appears to be lower in convalescents than in naïve individuals56. Pfizer's vaccine could reshape the innate immune response to other pathogens. It would induce immune tolerance that could either be beneficial in Covid-19 characterized by hyperinflammation or be deleterious by decreasing the response to other viral infections [and cancers]. This vaccine could also interfere with other vaccinations such as influenza, which is likely to be given at the same time in early fall57.

This reprogramming of immune immunity could be long-lasting due to epigenetic phenomena: "Training" of the innate immune response plays an important role both in diseases where host defense is impaired, such as cancers, and in autoinflammatory and autoimmune diseases, in which an exacerbated "trained" immunity phenotype could contribute to the pathogenesis of the disease. The impact of immune training, and more generally of epigenetic rewiring in the various priming, adaptation, or tolerance processes during disease, deserves further study. "58

\section{Conclusion}

Natural immunity to Covid-19 (i.e., obtained after natural infection with the virus) is therefore certainly robust and durable.

Work on post-vaccination immunity is mainly concerned with so-called "neutralizing" antibodies in vitro. The levels of these antibodies may not be a good correlate of protection because studies often find higher levels of antibodies after vaccination than after infection. However, reinfections are much more frequent in vaccinated patients than in convalescents. Protection against Covid-19 could rather depend on immune memory (due to memory $\mathrm{T}$ and $\mathrm{B}$ cells that persist long after infection) and seems to be of better quality than that conferred by vaccines.

In addition, vaccination of convalescent subjects could be risky: more systemic adverse events are observed in convalescent subjects than in naïve subjects after the first dose of vaccine.

Vaccination may decrease the ability to respond to future variants. It could also have a non- specific effect of remodeling the innate immune response by decreasing the potential response to other viruses or to cancers and by modifying the course of inflammatory and autoimmune diseases.

\section{References}

1 Tsakou-Ngouafo, L., Paganini, J., Kaufman, J. and Pontarotti, P. (2020). Origins of the RAG transposome and the MHC. Trends Immunol. 41(7),561-571. https://doi.org/10.1016/j.it.2020.05.002

2 (https://publichealth.jhu.edu/2021/what-is-herd-immunity-and-how-can-we-achieve-it-with-covid-19 Rethinking Herd Immunity and the Covid-19 Response End Game

3 The time course of the immune response to experimental coronavirus infection of man. https://www.ncbi.nlm.nih.gov/pmc/articles/PMC2271881/ )

4 (Ng et al., 2016) Ng OW, Chia A, Tan AT, Jadi RS, Leong HN, Bertoletti A, Tan YJ. Memory T cell responses 
targeting the SARS coronavirus persist up to 11 years post-infection. Vaccine. 2016 Apr 12;34(17):2008-14. doi: 10.1016/j.vaccine.2016.02.063. Epub 2016 Mar 5. PMID: 26954467; PMCID: PMC7115611

5 (Le Bert N, Tan AT, Kunasegaran K, Tham CYL, Hafezi M, Chia A, Chng MHY, Lin M, Tan N, Linster M, Chia WN, Chen MI, Wang LF, Ooi EE, Kalimuddin S, Tambyah PA, Low JG, Tan YJ, Bertoletti A. SARS- CoV-2specific T cell immunity in cases of COVID-19 and SARS, and uninfected controls. Nature. 2020 Aug;584(7821):457-462. doi: 10.1038/s41586-020-2550-z. Epub 2020 Jul 15. PMID: 32668444.) 6 [Huang AT, Garcia-Carreras B, Hitchings MDT, Yang B, Katzelnick LC, Rattigan SM, et al. A systematic review of antibody mediated immunity to coronaviruses: kinetics, correlates of protection, and association with severity. Nat Commun 2020;11:4704., https://www.nature.com/articles/s41467-020-18450-4]

7 [Dan JM, Mateus ], Kato Y, Hastie KM, Yu ED, Faliti CE, et al. Immunological mem- ory to SARS-CoV-2 assessed for up to 8 months after infection. Science 2021;371.].

8 Cho, Alice, Frauke Muecksch, Dennis Schaefer-Babajew, Zijun Wang, Shlomo Finkin, Christian Gaebler, Victor Ramos, et al. « Anti- SARS-CoV-2 Receptor Binding Domain Antibody Evolution after MRNA Vaccination », 30 août 2021. https://doi.org/10.1101/2021.07.29.454333 .

9 (Feng, Chengqian, Jingrong Shi, Qinghong Fan, Yaping Wang, Huang Huang, Fengjuan Chen, Guofang Tang, et al. «Protective Humoral and Cellular Immune Responses to SARS-CoV-2 Persist up to 1 Year after Recovery ». Nature Communications 12, no 1 (17 août 2021): 4984. https://doi.org/10.1038/s41467-02125312-0.)

10 Large-scale study of antibody titer decay following BNT162b2 mRNA vaccine or SARS-CoV-2 infection, https://www.medrxiv.org/content/10.1101/2021.08.19.21262111v1

11 (CD8+ T cell responses in COVID-19 convalescent individuals target conserved epitopes from multiple prominent SARS-CoV-2 circulating variants https://pubmed.ncbi.nlm.nih.gov/33594378/).

12 (Superior cellular and humoral immunity toward SARS-CoV-2 reference and alpha and beta VOC strains in COVID-19 convalescent as compared to the prime boost BNT162b2 vaccinated dialysis patients, https://www.kidney-international.org/article/S0085-2538(21)00680-3/abstract 13 Longitudinal analysis shows durable and broad immune memory after SARS-CoV-2 infection with persisting antibody responses and memory B and T cells, https://www.cell.com/cell-reportsmedicine/abstract/S2666- 3791(21)00203-2

14 Landscape of epitopes targeted by T cells in 852 individuals recovered from COVID-19: Meta-analysis, immunoprevalence, and web platform, https://www.sciencedirect.com/science/article/pii/S2666379121001555 15 The longitudinal kinetics of antibodies in COVID-19 recovered patients over 14 months, https://www.medrxiv.org/content/10.1101/2021.09.16.21263693v1, 16 BMJ : (Vaccinating people who have had covid-19: why doesn't natural immunity count in the US? BMJ 2021; 374 doi: https://doi.org/10.1136/bmj.n2101 (Published 13 September 2021

17 (High Seroprevalence of SARS-CoV-2 Eight Months After Introduction in Nairobi, Kenya, https://papers.ssrn.com/abstract $=3844795$ 
18 (SARS-CoV-2 seroprevalence among the general population and healthcare workers in India, December 2020- January 2021, 10.1016/j.ijid.2021.05.040)

19 Xu, X., Sun, J., Nie, S. et al. Seroprevalence of immunoglobulin M and G antibodies against SARS-CoV-2 in China. Nat Med 26, 1193-1195 (2020), https://doi.org/10.1038/s41591-020-0949-6

20 (https://www.has-sante.fr/jcms/p_3269831/fr/covid-19-des-trod-pour-optimiser-I-utilisation-des-dosesde- vaccins-disponibles

21 (Matthieu Schoenhals, Niry Rabenindrina, Jean Marius Rakotondramanga, Philippe Dussart, Rindra Randremanana, et al.. SARS-CoV-2 antibody seroprevalence follow-up in Malagasy blood donors during the 2020 COVID-19 Epidemic. EBioMedicine, Elsevier, 2021, 68, pp.103419. (10.1016/j.ebiom.2021.103419). (pasteur-03254611))

22 (The Role of Antibody Testing for SARS-CoV-2: Is There One?, https://pubmed.ncbi.nlm.nih.gov/32350047/)

23 Banoun H., 2020, COVID19: Cross-Immunity with Other Coronaviruses, Immunopathological Phenomena, http://ssrn.com/abstract=3654264

24 Highly functional virus-specific cellular immune response in asymptomatic SARS-CoV-2 infection, https://pubmed.ncbi.nlm.nih.gov/33646265/

25 https://www.cdc.gov/mmwr/volumes/70/wr/pdfs/mm7032e1-H.pdf Reduced Risk of Reinfection with SARS-

CoV-2 After COVID-19 Vaccination - Kentucky, May-June 202126 Correlates of protection from SARS-CoV2 infection

https://www.thelancet.com/journals/lancet/article/PIIS0140-6736(21)00782-0/fulltext

27 (Technical Briefing UK, July 23, 2021

https://assets.publishing.service.gov.uk/government/uploads/system/uploads/attachment_data/file/1005517/ Tech nical_Briefing_19.pdf.

28 (Gallais et al., Gallais, Floriane, Pierre Gantner, Timothée Bruel, Aurélie Velay, Delphine Planas, MarieJosée Wendling, Sophie Bayer, et al. «Evolution of Antibody Responses up to 13 Months after SARS-CoV-2 Infection and Risk of Reinfection ». EBioMedicine 71 (1 septembre 2021).

https://doi.org/10.1016/j.ebiom.2021.103561.)

29 SARS-CoV-2 seropositivity and subsequent infection risk in healthy young adults: a prospective cohort study,

https://www.thelancet.com/journals/lanres/article/PIIS2213-2600(21)00158-2/fulltext 30 https://wwwnc.cdc.gov/eid/article/27/10/21-1427_article publié 21 juillet 2021 par le CDC Breakthrough Infections of SARS-CoV-2 Gamma Variant in Fully Vaccinated Gold Miners, French Guiana, 2021

31 (Gazit, Sivan, Roei Shlezinger, Galit Perez, Roni Lotan, Asaf Peretz, Amir Ben-Tov, Dani Cohen, Khitam Muhsen, Gabriel Chodick, et Tal Patalon. « Comparing SARS-CoV-2 Natural Immunity to Vaccine-Induced Immunity: Reinfections versus Breakthrough Infections », 25 août 2021. 
https://doi.org/10.1101/2021.08.24.21262415.

32 , Having SARS-CoV-2 once confers much greater immunity than a vaccine-but no infection parties, please,

https://www.sciencemag.org/news/2021/08/having-sars-cov-2-once-confers-much-greater-immunityvaccine-no- infection-parties

33 Equivalency of Protection from Natural Immunity in COVID-19 Recovered Versus Fully Vaccinated Persons: A Systematic Review and Pooled Analysis,

https://www.medrxiv.org/content/10.1101/2021.09.12.21263461v1

34 (ChenX,ChenZ,AzmanAS,SunR,LuW,ZhengN,ZhouJ,WuQ,DengX,ZhaoZ,ChenX,GeS,Yang J, Leung DT, Yu $\mathrm{H}$. Neutralizing antibodies against SARS-CoV-2 variants induced by natural infection or vaccination: a systematic review and pooled meta-analysis. Clin Infect Dis. 2021 Jul 24:ciab646. doi: 10.1093/cid/ciab646. Epub ahead of print. PMID: 34302458

35 Hybrid immunity, https://science.sciencemag.org/content/372/6549/1392

36 mRNA vaccination boosts cross-variant neutralizing antibodies elicited by SARS-CoV-2 infection

L. Stamatatos et al., Science 372, 1413 (2021). https://pubmed.ncbi.nlm.nih.gov/33766944/

37 Prior SARS-CoV-2 infection rescues $B$ and $T$ cell responses to variants after first vaccine dose

C. J. Reynolds et al., Science 372, 1418 (2021) https://www.science.org/doi/10.1126/science.abh1282 38 Neutralization of VOCs including Delta one year post COVID-19 or vaccine, https://www.medrxiv.org/content/10.1101/2021.08.12.21261951v1

39 Reduced sensitivity of SARS-CoV-2 variant Delta to antibody neutralization https://www.nature.com/articles/s41586-021-03777-9

40 « Séroneutralisation SARS-CoV-2 : effets sur les variants » https://youtu.be/nZnzNCgvNq8

41(

CoV-2 https://doi.org/10.21203/rs.3.rs-783298/v2 La Scola

42 The role of antibodies in the light of the theory of evolution.

African Journal of Biological Sciences. 3(3), 1-9.

https://www.afjbs.com/files/1627021759_(1)_AFJBS20210145_(p_1-9).pdf

43 SARS-CoV-2-specific CD8<sup >+</sup > T cell responses in convalescent COVID-19 individuals, https://www.jci.org/articles/view/145476\#SEC3

44 SARS-CoV-2 specific memory B cells frequency in recovered patient remains stable while antibodies decay over time,

https://www.researchgate.net/publication/343867921_SARS-CoV-

2_specific_memory_B_cells_frequency_in_recovered_patient_remains_stable_while_antibodies_decay_over_t i me

45 (An infectivity-enhancing site on the SARS-CoV-2 spike protein targeted by antibodies https://www.cell.com/cell/abstract/S0092-8674(21)00662-0, Enhancing anti-SARS-CoV-2 antibodies recognize both the original Wuhan/D614G strain and Delta variants. A potential risk for mass vaccination ?, 
Journal of Infection (2021), doi: https://doi.org/10.1016/j.jinf.2021.08.010, The SARS-CoV-2 Delta variant is poised to acquire complete resistance to wild-type spike vaccines

https://www.biorxiv.org/content/10.1101/2021.08.22.457114v1, The plasmablast response to SARS-CoV-2 mRNA vaccination is dominated by non-neutralizing antibodies that target both the NTD and the RBD https://www.medrxiv.org/content/10.1101/2021.03.07.21253098v1

46 Mucosal Immunity in COVID-19: A Neglected but Critical Aspect of SARS-CoV-2 Infection, https://www.frontiersin.org/articles/10.3389/fimmu.2020.611337/full

47 mRNA vaccine-induced T cells respond identically to SARS-CoV-2 variants of concern but differ in longevity and homing properties depending on prior infection status,

https://www.biorxiv.org/content/10.1101/2021.05.12.443888v2

48 Identification of resident memory CD8+ T cells with functional specificity for SARS-CoV-2 in unexposed oropharyngeal lymphoid tissue, https://www.science.org/doi/10.1126/sciimmunol.abk0894 49 What's causing long COVID?,16 August 2021, Interview with Akiko Iwasaki, Yale University https://www.thenakedscientists.com/articles/interviews/whats-causing-long-covid 50 Neuro-COVID long-haulers exhibit broad dysfunction in T cell memory generation and responses to vaccination https://www.medrxiv.org/content/10.1101/2021.08.08.21261763v2 51 (Antibody Responses in Seropositive Persons after a Single Dose of SARS-CoV-2 mRNA Vaccine I NEJM,https://www.nejm.org/doi/full/10.1056/NEJMc2101667, Life | Free Full-Text | Self-Reported Real-World Safety and Reactogenicity of COVID-19 Vaccines: A Vaccine Recipient Survey | HTML, https://www.mdpi.com/2075-1729/11/3/249/htm, Previous COVID-19 infection but not Long-COVID is associated with increased adverse events following BNT162b2/Pfizer vaccination, https://www.medrxiv.org/content/10.1101/2021.04.15.21252192v1, Robust spike antibody responses and increased reactogenicity in seropositive individuals after a single dose of SARS-CoV-2 mRNA vaccine | medRxiv, https://www.medrxiv.org/content/10.1101/2021.01.29.21250653v1.full, Safety and humoral responses to BNT162b2 mRNA vaccination of SARS-CoV-2 previously infected and naive populations | Scientific Reports, https://www.nature.com/articles/s41598-021-96129-6, Vaccine side-effects and SARSCoV-2 infection after vaccination in users of the COVID Symptom Study app in the UK: a prospective observational study, https://www.thelancet.com/journals/laninf/article/PIIS1473-3099(21)00224-3/fulltext). 52 Naturally enhanced neutralizing breadth against SARS-CoV-2 one year after infection, http://www.nature.com/articles/s41586-021-03696-9 53 Differential effects of the second SARS-CoV-2 mRNA vaccine dose on T cell immunity in naïve and COVID- 19 recovered individuals https://www.biorxiv.org/content/10.1101/2021.03.22.436441v1) High individual heterogeneity of neutralizing activities against the original 4 strain and 9 different variants of

\section{SARS-}

54 https://www.biorxiv.org/content/10.1101/2021.07.29.454333v2

Anti- SARS-CoV-2 Receptor Binding Domain Antibody Evolution after mRNA Vaccination 
55 Horndler, Lydia, Pilar Delgado, Salvador Romero-Pinedo, Marina Quesada, Ivaylo Balabanov, Rocío Laguna- Goya, Patricia Almendro-Vázquez, et al. « DECREASED BREADTH OF THE ANTIBODY RESPONSE TO THE SPIKE PROTEIN OF SARS-CoV-2 AFTER VACCINATION », 14 août 2021.

https://doi.org/10.1101/2021.08.12.21261952

56 mRNA vaccine-induced T cells respond identically to SARS-CoV-2 variants of concern but differ in longevity and homing properties depending on prior infection status, https://www.biorxiv.org/content/10.1101/2021.05.12.443888v2

57 The BNT162b2 mRNA vaccine against SARS-CoV-2 reprograms both adaptive and innate immune responses,

https://www.medrxiv.org/content/10.1101/2021.05.03.21256520v1

58 https://www.nature.com/articles/s41577-020-0285-6, Defining trained immunity and its role in health and disease 